\title{
Symptomatic Temporomandibular Joint Herniation through the Foramen of Huschke: A Case Report
}

\author{
Hee-Jeong Song ${ }^{1}$, Yuni Lee ${ }^{2}$, Hang-Moon Choi ${ }^{3}$, Young-Jun Kim ${ }^{1}$, Cheul Kim ${ }^{1}$, Moon-Soo Park ${ }^{1}$ \\ 1Department of Oral Medicine and Diagnosis, Research Institute of Oral Science, College of Dentistry, \\ Gangneung-Wonju National University, Gangneung, Korea \\ ${ }^{2}$ Department of Periodontology, College of Dentistry, Gangneung-Wonju National University, Gangneung, Korea \\ ${ }^{3}$ Department of Oral and Maxillofacial Radiology, Research Institute of Oral Science, College of Dentistry, \\ Gangneung-Wonju National University, Gangneung, Korea
}

Received November 26, 2021

Revised December 9, 2021

Accepted December 9, 2021
Correspondence to:

Moon-Soo Park

Department of Oral Medicine and Diagnosis, College of Dentistry, Gangneung-Wonju

National University, 7 Jukheon-gil,

Gangneung 25457, Korea

Tel: $+82-33-640-2466$

Fax: +82-33-640-3129

E-mail:mpark@gwnu.ac.kr

https://orcid.org/0000-0002-3076-0647
The foramen of Huschke (FH) or foramen tympanicum is a persistent bony defect connecting the external auditory canal (EAC) to the temporomandibular joint (TMJ). It arises from an incomplete ossification of the tympanic part of the temporal bone that persists after the age of 5. If a herniation exists in the TMJ, otological symptoms may occur. An 80-year-old female patient complained of noise in her left TMJ and otorrhea in her left ear. On her cone beam computed tomography images, there were only degenerative joint disease signs on her left mandibular condyle. However, her computed tomography images revealed that the soft tissue of the TMJ herniate into the EAC. Additional examination was planned for the further evaluation. But the patient no longer visited the hospital due to her systemic health status, and symptoms disappeared spontaneously without any treatments. Usually this type of herniation is very rare, but years of mechanical stress from mastication may result in weakening and widening of the foramen with age. Therefore, although FH is usually congenital, sometimes it may be acquired in the elderly. The treatment plan should be determined in consideration of the patient's symptom level and the patient's general health status. If the clinical symptoms are not severe, no treatment is required.

Key Words: External auditory canal; Foramen of Huschke; Herniation

\section{INTRODUCTION}

Temporomandibular disorders (TMDs) are a collective term that includes a wide range of abnormalities occurring in the muscles and joints in the maxillofacial region, and mainly exhibits features such as pain in the maxillofacial region, temporomandibular joint (TMJ) noises, and changes or disorders in jaw function. TMD can be divided into TMJ disorders and masticatory muscle disorders. TMJ disorders include morphological changes, internal derangement, inflammatory conditions, arthritis and joint ankylosis, and masticatory muscle disorders include myofascial pain, myositis, muscle spasms, protective muscle co-contraction, muscle contracture and neoplasm [1,2].

TMD are challenging to diagnose because they can cause many different non-specific symptoms. These may include, but are not limited to, headache, dizziness, paresthesia, and otological symptoms. Among non-specific symptoms, some symptoms are otological manifestations such as otalgia, tinnitus, vertigo, ear pruritus, and a feeling of fullness of the ear [3-5]. The external auditory canal (EAC) is intimately related to the TMJ, separated only by its bony anterior wall, which is of variable thickness. The foramen of Huschke (FH) represents a developmental defect in the antero-inferior 
aspect of the bony external auditory meatus and is an uncommon disorder [6,7]. The foramen is located at the antero-inferior aspect of EAC, postero-medial to TMJ. In general, FH is asymptomatic, but if symptoms are present, they are usually otological symptoms and these symptoms may appear as a result of TMJ herniation through FH $[6,8]$.

The authors report a case of symptomatic TMJ herniation through $\mathrm{FH}$, and discuss the evaluation of TMD patients with otological symptoms. The study was approved by the Institutional Review Board of GangneungWonju National University Dental Hospital (IRB no. GWNUDH-IRB2021-A013).

\section{CASE REPORT}

An 80-year-old female presented with complaints of noise in her left TMJ and otorrhea in her left ear. She had been taken a computed tomography (CT) scan in the otorhinolaryngology clinic beforehand, and was then referred to our hospital. She suffered from hypertension, diabetes and arthritis, and was wearing in-the-ear hearing aids due to hearing loss.

Six months ago, she had been diagnosed with the degenerative joint disease (DJD) of left TMJ in our hospital, but there was no specific finding on cone beam computed tomography (CBCT) except for DJD signs at that time. On her clinical examination, there were no specific findings related to her chief complaints, except for the crepitus of left TMJ. Through the re-evaluation of the previous CT images, we found a polypoid mass with a diameter of about $1 \mathrm{~cm}$ in the antero-inferior wall of the left EAC and osseous defects in that area (Fig. 1). We also re-checked the CBCT images taken in our hospital 6 months earlier than the CT images. FH was confirmed, but a definite TMJ soft tissue herniation could not be found (Fig. 2).

Based on the above findings, the clinical impression was
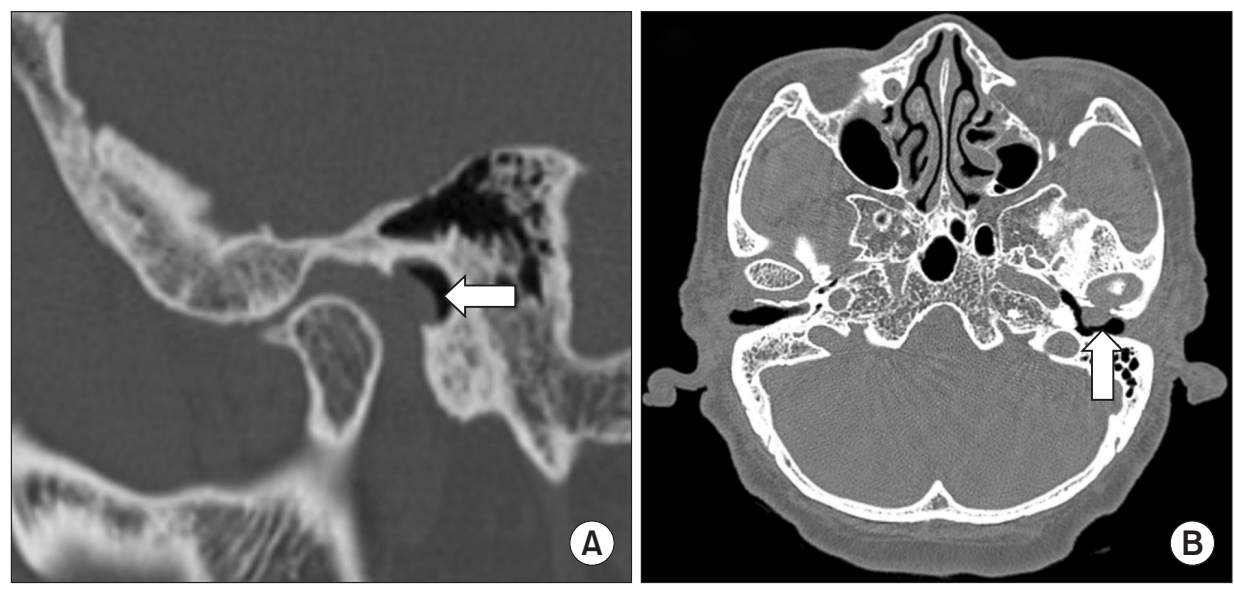

Fig. 1. Closed-mouth computed tomography image showing defect (arrow) in antero-inferior wall of external auditory canal (EAC) with protrusion of the temporomandibular joint soft tissue into EAC: $(A)$ sagittal view; (B) axial view.
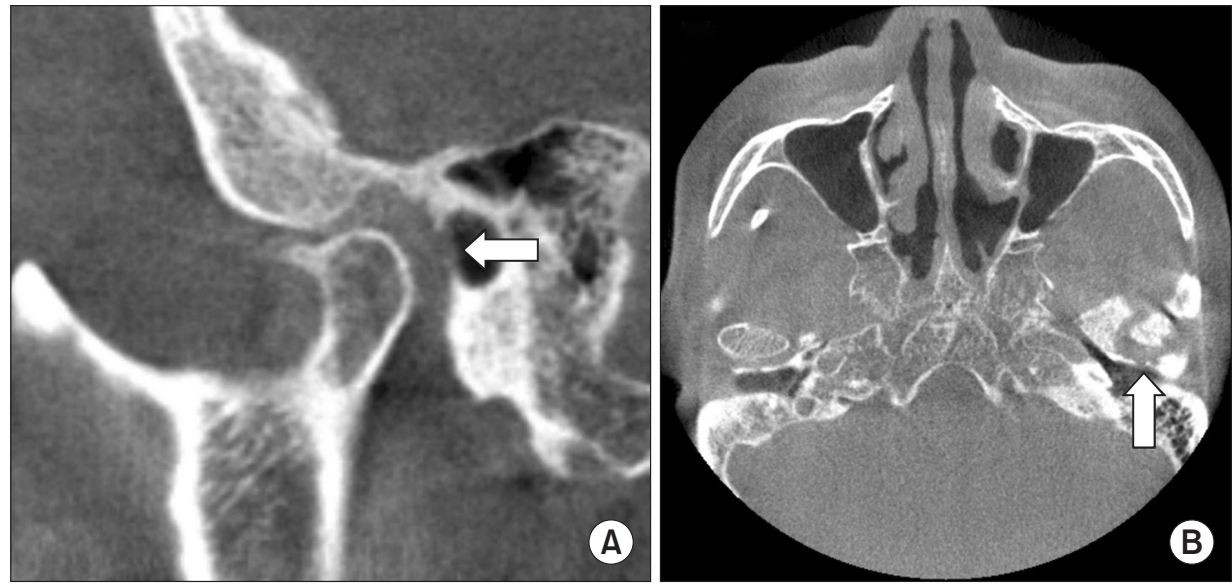

Fig. 2. Closed-mouth cone beam computed tomography image showing defect (arrow) in antero-inferior wall of external auditory canal: $(A)$ sagittal view; (B) axial view. 
made as TMJ herniation through $\mathrm{FH}$, and enhanced CT was planned for the further evaluation. However, additional CT examination could not be performed because she did not return to our hospital due to her systemic health problem.

Several weeks later, the patient's condition was confirmed through a phone call with her guardian, and he said that there was no swelling and otorrhea in her left ear. In addition, we informed that there was no pain related to TMJ DJD except for mild crepitus during mouth opening.

\section{DISCUSSION}

Spontaneous herniation of TMJ has been attributed to persistence of the FH. Even if FH is present, it is rare for TMJ soft tissue to protrude into the EAC and to cause clinical symptoms such as otalgia, tinnitus, vertigo, ear pruritus, and a feeling of fullness of the ear. It was reported that FH was detected in 137 (13.4\%) of 1,025 patients with otological symptoms. The prevalence of FH was higher in females than in males and the presence of FH increased with age. Herniation of the TMJ through FH into the EAC was seen in $0.4 \%$ of patients, and 2.9\% of patients with FH had TMJ herniation into EAC [9].

Another study found that the average age of patients with TMJ herniation through FH was 55 years, and more than $80 \%$ of patients were 50 years or older. The authors of this study presented the long-term excessive mastication as an etiology. In other words, the mechanical overloading caused by masticatory function for many years can induce the decrease in the thickness of the anterior auditory meatus wall and the increase in the size of the FH with increasing age. Therefore, it can be inferred that the size of FH may increase due to the masticatory force with age [10]. Since clenching is a commonly observed parafunction in TMD patients, there is a possibility that FH can be commonly found in TMD patients. Therefore, future studies on the prevalence of FH in TMD patients should be performed.

Treatment is not necessary unless clinical symptoms are present, and it is recommended that the severity of clinical symptoms and the condition of the patient should be taken into consideration. Conservative or surgical approaches are possible. First, conservative treatment such as medications and behavioral modifications for reducing masticatory force can be used for patients with mild symptoms [10,11]. With a surgical approach, the bone defect in EAC can be closed with certain material. This can be applied when the clinical symptoms are severe or the patient has a strong will to solve them with surgery, and has the advantage that clinical symptoms can be clearly improved. Usually, surgical procedure is performed by incision using a preauricular or endoural approach, but the former method is advantageous for TMJ reconstruction. Tragal cartilage graft and polyethylene, polypropylene, or titanium miniplate can be used to close the defect. But short-term symptom relief has been reported in only several studies, and studies on the long-term prognosis are still insufficient [11].

In case of polypoid mass infiltrating EAC from TMJ on CT image, we should consider the possibility of soft tissue tumors in the joint capsule, cholesteatoma in EAC, and ear trauma in addition to TMJ herniation. It is known that tumors within the joint capsule are extremely rare [12], and TMJ tumors usually show preauricular swelling. But in this case, no swelling was observed, therefore tumors could be excluded. However, if enhanced CT was taken, it would be helpful to rule out the tumor. EAC choleastoma is characterized by bone erosion inside the soft tissue mass or intramural bone fragments [13], and these findings were not observed in this case. Since all the bones around the foramen were connected, the possibility of bone fracture could also be ruled out. The cause of bone fractures constituting EAC is usually trauma to the mandible or temporal bone [14], and this patient had no history of such trauma. Otorrhea can occur when TMJ herniation is damaged by an external irritation. In this case, it is speculated that in-the-ear hearing aids worn by the patient may have continuously compressed the TMJ herniation.

In this study, we have reported a case of TMJ herniation through the FH presenting with otorrhea. When evaluating elderly TMD patients with non-specific symptoms related to the ears, the presence of FH and the possibility of TMJ herniation through it should be considered.

\section{CONFLICT OF INTEREST}

No potential conflict of interest relevant to this article was reported. 
ORCID

\author{
Hee-Jeong Song \\ https://orcid.org/0000-0002-8787-1735 \\ Yuni Lee \\ https://orcid.org/0000-0002-8584-1786 \\ Hang-Moon Choi \\ https://orcid.org/0000-0003-1222-1348 \\ Young-Jun Kim \\ https://orcid.org/0000-0003-2866-5517 \\ Cheul Kim \\ https://orcid.org/0000-0001-7935-3244 \\ Moon-Soo Park \\ https://orcid.org/0000-0002-3076-0647
}

\section{REFERENCES}

1. Liu F, Steinkeler A. Epidemiology, diagnosis, and treatment of temporomandibular disorders. Dent Clin North Am 2013;57:465479.

2. Ohrbach R, Dworkin SF. The evolution of TMD diagnosis: past, present, future. J Dent Res 2016;95:1093-1101.

3. Tuz HH, Onder EM, Kisnisci RS. Prevalence of otologic complaints in patients with temporomandibular disorder. Am J Orthod Dentofacial Orthop 2003;123:620-623.

4. Kusdra PM, Stechman-Neto J, Leão BLC, Martins PFA, Lacerda ABM, Zeigelboim BS. Relationship between otological symptoms and TMD. Int Tinnitus J 2018;22:30-34.

5. Porto De Toledo I, Stefani FM, Porporatti AL, et al. Prevalence of otologic signs and symptoms in adult patients with temporomandibular disorders: a systematic review and meta-analysis. Clin Oral Investig 2017;21:597-605.

6. van der Meer WL, van Tilburg M, Mitea C, Postma AA. A persistent foramen of Huschke: a small road to misery in necrotizing external otitis. AJNR Am J Neuroradiol 2019;40:1552-1556.

7. Soudry E, Hamzany Y, Preis M, Joshua B, Hadar T, Nageris BI. Malignant external otitis: analysis of severe cases. Otolaryngol Head Neck Surg 2011;144:758-762.

8. Glikson E, Sagiv D, Wolf M, Shapira Y. Necrotizing otitis externa: diagnosis, treatment, and outcome in a case series. Diagn Microbiol Infect Dis 2017;87:74-78.

9. Ertugrul S, Keskin NK. Relationship of age to foramen of Huschke and investigation of the development of spontaneous temporomandibular joint herniation. Int J Oral Maxillofac Surg 2019;48:534-539.

10. Moriyama M, Kodama S, Suzuki M. Spontaneous temporomandibular joint herniation into the external auditory canal: a case report and review of the literature. Laryngoscope 2005;115:21742177.

11. Lim KH, Jung JY, Rhee J, Choi J. Temporomandibular joint herniation through the foramen of Huschke with clicking tinnitus. Eur Ann Otorhinolaryngol Head Neck Dis 2019;136:497-499.

12. Mallya S, Lam E. White and pharoah's oral radiology: principles and interpretation. 8th ed. St. Louis: Elsevier; 2019. pp. 602-604.

13. Heilbrun ME, Salzman KL, Glastonbury CM, Harnsberger HR, Kennedy RJ, Shelton C. External auditory canal cholesteatoma: clinical and imaging spectrum. AJNR Am J Neuroradiol 2003;24:751-756.

14. Burchhardt DM, David J, Eckert R, Robinette NL, Carron MA, Zuliani GF. Trauma patterns, symptoms, and complications associated with external auditory canal fractures. Laryngoscope 2015;125:1579-1582. 